\title{
Pharmacological Properties of Shikonin - A Review of Literature since 2002
}

Authors

Affiliation
Isabel Andújar, José Luis Ríos, Rosa María Giner, María Carmen Recio

Departament de Farmacologia, Facultat de Farmàcia, Universitat de València, Valencia, Spain
Key words

- anti-infectious

- anti-inflammatory

- antioxidant

- glucose metabolism

- wound healing

- shikonin

- Lithospermum erythrorhizon

- Boraginaceae

\section{Abstract}

$\nabla$

The naphthoquinone shikonin is the main active principle of Zicao, a traditional Chinese herbal medicine made from the dried root of Lithospermum erythrorhizon. Studies carried out over the past 30 years have provided a scientific basis for the use of Zicao which has been long employed in folk medicine to treat a variety of inflammatory and infectious diseases. In particular, shikonin has been shown to possess many diverse properties, including antioxidant, anti-inflammatory, antithrombotic, antimicrobial, and wound healing effects. The fact that shikonin shows so many beneficial properties has increased the interest in this molecule dramatically, especially in the past few years. The aim of this review is to provide an update of the new data published on shikonin, whose wide spectrum of pharmacological effects as well as pharmacokinetic properties and toxicity make it a highly interesting target molecule.

$\begin{array}{ll}\text { received } & \text { May 23, } 2013 \\ \text { revised } & \text { July 29, 2013 } \\ \text { accepted } & \text { Sept. 16, 2013 }\end{array}$

Bibliography

DOI http://dx.doi.org/

10.1055/s-0033-1350934

Published online October 23,

2013

Planta Med 2013; 79:

1685-1697 @ Georg Thieme

Verlag KG Stuttgart · New York . ISSN 0032-0943

\section{Correspondence}

Prof. Dr. María Carmen Recio Departament de Farmacologia

Facultat de Farmàcia

Universitat de València

Av. Vicent Andrés Estellés s/n

46100 Burjassot, Valencia

Spain

Phone: + 34963543283

Fax: + 34963544943

maria.c.recio@uv.es
c-Maf: $\quad$ c-musculoaponeurotic fibrosarcoma

COX: cyclooxygenase

DPPH: 1,1-diphenyl-2-picryl-hydrazyl

$\mathrm{EC}_{50}$ : $\quad$ effective concentration 50

ERK: extracellular signal-regulated kinase

GLUT4: $\quad$ glucose transporter type 4

HCV: $\quad$ hepatitis $C$ virus

HIV: human immunodeficiency virus

$\mathrm{IC}_{50}$ : inhibitory concentration 50

I $k$ B: $\quad$ inhibitor of NF- $k$ B

IL: interleukin

iNOS: $\quad$ inducible nitric oxide synthase

$\mathrm{LC}_{50}$ : lethal concentration 50

LDL: low-density lipoprotein

LPS: lipopolysaccharide

LRS: lactated Ringer's solution

MIC: minimal inhibitory concentration

MPO: myeloperoxidase

NAT: $\quad N$-acetyltransferase

NF- $k$ B: $\quad$ nuclear factor $k B$

PPAR: $\quad$ peroxisome proliferator-activated receptor

RIP: receptor interacting protein

ROS: reactive oxygen species

SREBP: $\quad$ sterol regulatory element-binding protein

STAT3: $\quad$ signal transducer and activator of transcription-3

TATA box-binding protein: transcription factor IID protein complex

T-bet: $\quad$ T-box expressed in T cells

TNF: $\quad$ tumor necrosis factor

TPA: 12-0-tetradecanoylphorbol-13acetate 


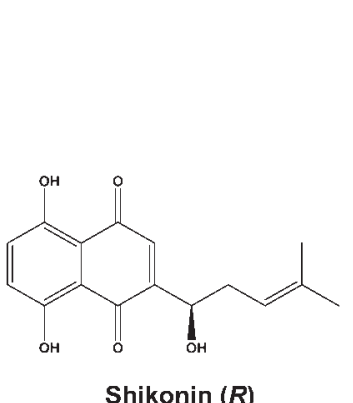<smiles>O=C1C=CC(=O)c2c(O)ccc(O)c21</smiles>
Naphthazarin<smiles>CC(C)=CC[C@@H](O)C1=CC(=O)c2c(O)ccc(O)c2C1=O</smiles>

Alkannin $(S)$<smiles>CC(C)=CCCC1=CC(=O)c2c(O)ccc(O)c2C1=O</smiles><smiles>CC(=O)O[C@H](CC=C(C)C)C1=CC(=O)c2c(O)ccc(O)c2C1=O</smiles>

Deoxyshikonin

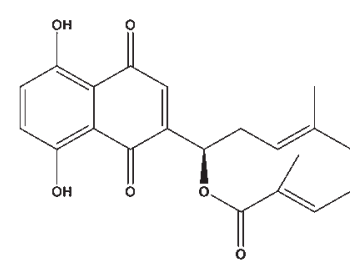

\section{Introduction}

\section{Sources of shikonin and pharmacological effects of} Lithospermum erythrorhizon extracts

Shikonin is one of the active principles of Zicao, the root extract of Lithospermum erythrorhizon Sieb. et Zucc. (Boraginaceae), widely used in traditional Chinese medicine for its anti-inflammatory properties [1]. The first derivative of this extract to be described was acetylshikonin, which was isolated from the root of L. erythrorhizon by Kuroda and Majima in 1922. These same chemists later isolated other derivatives, including shikonin, and identified many of their chemical properties. However, because of its physical and chemical similarities to naphthazarin, Kuroda and Majima's original description of shikonin's structure was inaccurate. Some years later, in 1936, Brockmann and Liebigs elucidated the correct structure of this molecule, which was identified as 5,8-dihydroxy-2-[(1R)-1-hydroxy-4-methyl-3-pentenyl]-1,4naphthoquinone ( $\odot$ Fig. 1 ). They were also the first to identify shikonin's enantiomer alkannin [2]. Although mostly found in the root of $L$. erythrorhizon, shikonin, along with alkannin and other derivatives, can also be found in the roots of other species of the Boraginaceae family. These species include those from the genera Arnebia (such as A. euchroma), Echium (E. lycopsis), Eritrichium, and Onosma, among others [2].

Studies carried out over the past three decades have demonstrated that many effects associated with the use of Zicao in Chinese medicine have a scientific basis, with shikonin and its derivatives being the principal substances responsible for the pharmacological properties of this crude drug.

Until 2002, only 86 articles on shikonin could be found in Pubmed. Since then, however, over 200 more articles have been published. This exponential increase in interest in shikonin may be motivated by the fact that this molecule has been demonstrated to have interesting pharmacological properties, including anti-inflammatory, anticancer, antimicrobial, and wound healing effects. This review, therefore, is focused on the current knowledge of this naphthoquinone, which, because of its wide spectrum of effects and numerous molecular targets, is a potentially highly interesting target molecule.
The pharmacokinetics and bioavailability of shikonin Based on the long history of Zicao as an effective treatment for a variety of inflammatory and infectious diseases, various studies have been carried out to identify its primary active principles, with all of them indicating that shikonin and its derivatives are the main active components [1]. Previous reviews [1,2] had already reported on the known pharmacological properties of shikonin as well as on its pharmacokinetic behavior and toxicity. Unfortunately, studies on the toxicity of this molecule in different species and with different administration routes are still missing and should be carried out.

Due to its high lipophilicity, shikonin is generally used in creams and ointments, that is, oil-based preparations; indeed, its insolubility in water is usually the cause of its low bioavailability [3]. Moreover, shikonin and its derivatives are also thermolabile and prone to oxidation and polymerization [3-5]. One study using ${ }^{3} \mathrm{H}$-shikonin in mice showed that shikonin was rapidly absorbed after oral and intramuscular administration, with a half-life in plasma of $8.79 \mathrm{~h}$ and a distribution volume of $8.91 \mathrm{~L} / \mathrm{kg}$. After intravenous injection, most of the excreted radioactivity corresponded to the transformed metabolite. In order to improve the stability and water solubility of shikonin, several approaches have been tried. Thus, in 1996, Chen et al. [6] were able to achieve an approximate 200-fold increase in the solubility, photostability, and in vitro permeability of shikonin through the formation of a $1: 1$ inclusion complex with hydroxypropyl- $\beta$-cyclodextrin. In 2004, Assimopoulou and Papageorgiou [5] confirmed these results in a study in which they explored the inclusion of shikonin, alkannin, and their derivatives in $\beta$-cyclodextrin and hydroxypropyl- $\beta$-cyclodextrin. More recently, Albreht et al. [3] were able to achieve a 181-fold increase in the solubility of shikonin in aqueous media in the presence of $\beta$-lactoglobulin at a concentration of $3.1 \mathrm{mg} / \mathrm{mL}$. Because they are generally recognized as safe, proteins such as $\beta$-lactoglobulin are widely used in the food and pharmaceutical industries. One shikonin molecule binds covalently to $\beta$-lactoglobulin via Cys121, whereas the remaining pigment molecules most probably bind to the protein via non-covalent interactions. In order to facilitate cellular intake, Xia et al. [7] prepared shikonin-containing liposomes using shikonin, soybean phospholipid and cholesterol, and dehydrated alcohol. The lipo- 
somes were used to study the angiogenic suppression and cellular uptake by the MTT assay, Transwell tests, as well as chick chorioallantoic membrane and Matrigel plug assays, which demonstrated that shikonin liposomes decreased toxicity, rate of inhibition of migration, and angiogenic suppression compared to free shikonin.

A different approach involves the encapsulation of shikonin. Assimopoulou et al. [8] found that the combination of ethylcellulose (viscosity $=10 \mathrm{cp}$ ) as a matrix, mastic gum as a core additive, low dichloromethane volume, and low sodium dodecyl sulfate concentration results in microcapsules with the best characteristics in terms of efficiency, loading, release, and particle size distribution. Huang et al. [9] studied different alternatives for microencapsulation and established the best proportions for stability and coacervation processes as a surfactant/oil ratio of $1 / 10$ and a gelatin/oil ratio of $1 / 5$ with a $\mathrm{pH}$ condition of 4-6. They also observed that one core microcapsule prepared with $6 \%$ glycerol could be a good potentially nontoxic crosslinking material for the applications of encapsulated extracts containing shikonin, because it has the same crosslinking effects as those used to obtain gelatin/acacia microcapsules.

Other biodegradable and/or biocompatible polymeric materials are the electrospun poly( $\varepsilon$-caprolactone)/poly(trimethylene carbonate) ultrafine composite fiber mats, prepared by Han et al. [10] who demonstrated their potential as effective carriers for shikonin delivery. After demonstrating the drug stability, high drug-loading efficacy, and the stability of the shikonin-loaded composite fibers, they studied the in vitro release and observed that the initial rapid release of shikonin is followed by a plateau after $11 \mathrm{~h}$. This release behavior could be used as a drug delivery system but also in the treatment of wound healing or dermal bacterial infections due to the free radical scavenging properties and the antibacterial activity (which will be later discussed in this review) of the shikonin-loaded fiber mats.

This interest in topical or transdermal biomaterial containing shikonin was later ratified by Kontogiannopoulos et al. [11]. They prepared different biocompatible polymers to produce electrospun fiber mats containing shikonin in various amounts. Shikonin was incorporated and did not affect fibers morphology. In addition, they conclude that the prepared shikonin-loaded e-spun submicron-sized fiber mats are advantageous compared to conventional wound healing devices. One year later, the same authors [12] used chi-aDDnSs, which are mixed nanosystems combining different biomaterials that can offer advantages as drug carriers. They applied this system for the characterization of the shikonin-loaded chi-aDDnSs, which was performed by measuring their particle size distribution, $\zeta$-potential, drug encapsulation efficiency, stability, and the in vitro release profile. These properties gave promising results, which confirmed the interest of this kind of formulations, and the authors hypothesize that it could be used as a road map for designing in vivo experiments.

\section{Shikonin in Inflammation}

$\nabla$

The anti-inflammatory effect of shikonin has been studied for years in animal models of inflammation, and its potential mechanisms have been investigated in vitro. The reviews published by Chen et al. [1] and Papageorgiou et al. [2] list the most relevant studies conducted up to 2002, concluding that the anti-inflammatory effects of shikonin and some of its derivatives can be attributed to several distinct mechanisms of action, for example, inhibition of the biosynthesis of leukotriene $\mathrm{B}_{4}$, suppression of mast cell degranulation, inhibition of the respiratory burst in neutrophils, alteration of phosphatidylinositol-mediated signaling, or blockade of chemokine binding to the CCR-1. According to Kourounakis et al. [13], the naphthazarin structure of shikonin and its derivatives makes them free radical scavengers. This may be one of the mechanisms of action that gives shikonin its antiinflammatory properties. It was also demonstrated that shikonin has a better potency as a COX inhibitor than alkannin, but also higher cytotoxicity and pro-oxidant activity [14]. However, recent papers have demonstrated the implication of other mediators as well as other signaling pathways, thus broadening shikonin's possible mechanisms of action and therapeutic potential.

\section{Shikonin in classic anti-inflammatory mouse models}

The dichloromethane extract of $L$. erythrorhizon was studied by Zeng [15] in two classic models of anti-inflammatory activity: mouse ear edema induced by application of TPA and mouse paw edema induced by subcutaneous injection of carrageenan into the plantar foot. In both cases, the extract was observed to inhibit the edema. The researchers identified the prenylnaphtoquinones present in this extract as the probable active agents. The activity of other shikonin derivatives as anti-inflammatories has been also established by other authors, such as Kundakovic et al. [16], who described the effects of $\beta, \beta$-dimethylacrylshikonin, isovalerylshikonin, and acetylshikonin on a carrageenan-induced rat paw edema test, the last one being the best anti-inflammatory agent, with an $\mathrm{ED}_{50}$ of $6.91 \mathrm{mg} / \mathrm{kg}$ (p.o.).

In addition, the $n$-hexane extract obtained from the roots of L. erythrorhizon, which contains shikonin, acetyl shikonin, isobutyryl shikonin, $\beta, \beta$-dimethylacryl shikonin, and isovaleryl shikonin as the most relevant compounds, was tested on the transcriptional activation of the TNF- $\alpha$ promoter in mouse abdominal skin transfected with plasmid pTNFP-Luc via gene gun delivery as a model of inflammation [17]. This hexane extract (100 $\mu \mathrm{g}$ of each test agent/site/mouse) significantly inhibited (49\%) the transcriptional activity of the TNF- $\alpha$ promoter in comparison with the untreated controls in the in vivo system. All the tested compounds inhibited TNF- $\alpha$ promoter activity in a dose-dependent manner, but shikonin (77-96\% inhibition at 50-200 $\mu \mathrm{g} / \mathrm{site} /$ mouse) and isobutyryl shikonin (61-89\%) exhibited the highest inhibition of the transcriptional activation of the human TNF- $\alpha$ promoter through interference with the basal transcription machinery, inhibiting the binding of transcription factor IID protein complex (TATA box-binding protein) to the TATA box. In contrast, shikonin did not inhibit the phosphorylation of ERK1/2 or NF- $k B$ p65 induced by particle-mediated injury/stress in mouse skin [17].

Other studies have been carried out using the active principle shikonin. Andújar et al. [18] studied the effect of shikonin on mouse ear edema induced by TPA and found that it exerted antiinflammatory activity, as demonstrated by the reduction of the edema, the inhibition of COX-2 by $70 \%$, and the complete inhibition of iNOS. Topical treatment with shikonin significantly decreased TPA-induced translocation of protein kinase $\mathrm{C} \alpha$ along with the phosphorylation and activation of ERK1/2, the nuclear translocation of NF- $k \mathrm{~B}$, and the TPA-induced NF- $k$ B-DNA-binding activity in mouse skin. These results were confirmed in vitro in RAW 264.7 cells, where shikonin $(1 \mu \mathrm{M})$ significantly inhibited the binding of NF- $k$ B to DNA in a dose-dependent manner as well as the nuclear translocation of p65 through a mechanism involving the downregulation of the inhibitor of NF- $k \mathrm{~B}(\mathrm{I} k \mathrm{~B}) \alpha$ phospho- 


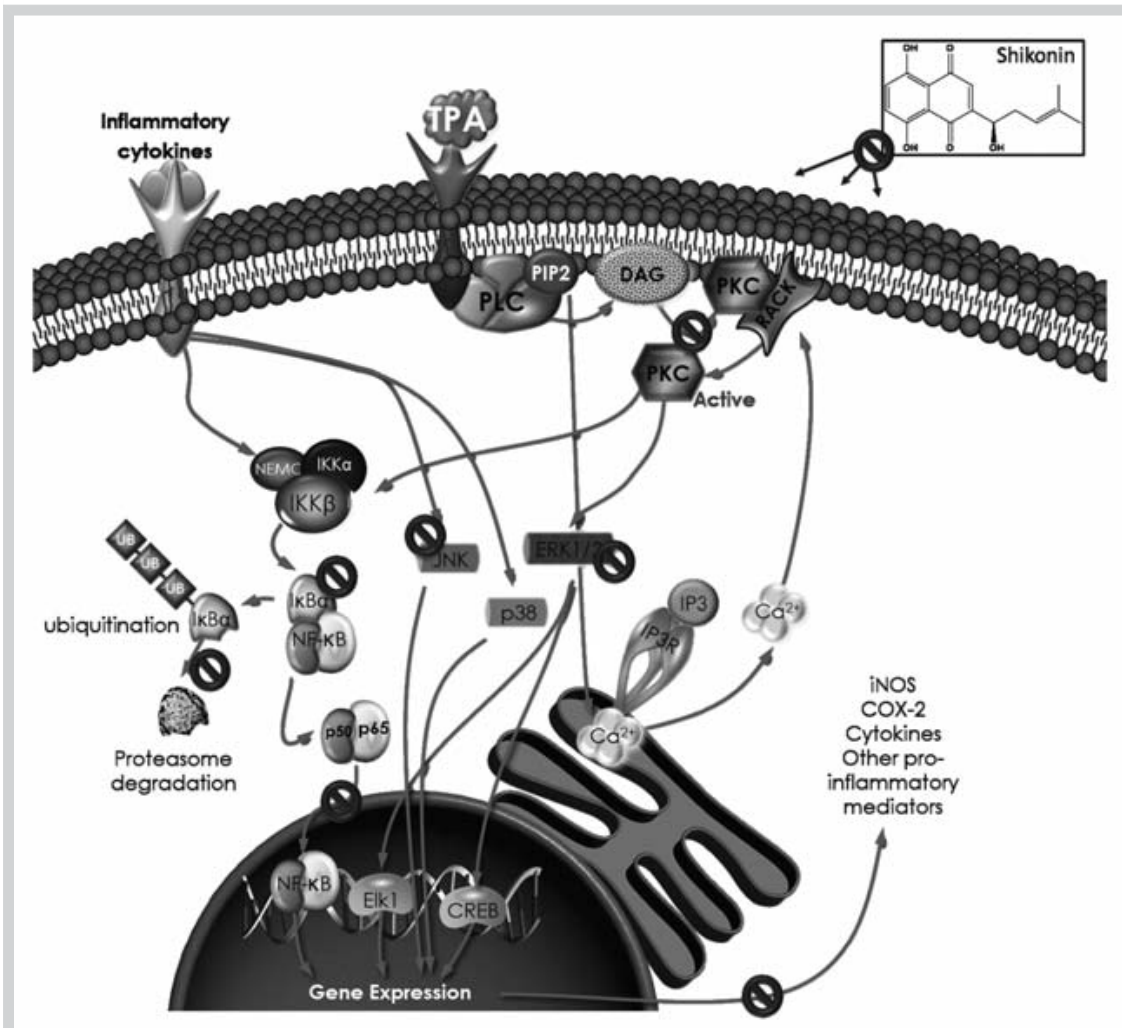

Fig. 2 Suggested targets for shikonin in inflammation. CREB (CAMP response element-binding protein); DAG (diacylglicerol); IKK (IKB kinase); IP3 (inositol trisphosphate); IP3R (inositol trisphosphate receptor); NEMO (NF-KB essential modulator); PIP2 (phosphatidylinositol 4,5-bisphosphate); PKC (protein kinase C); PLC (phospholipase C); RACK (receptor for activated C-kinase).

rylation. In a complementary study, Cheng et al. [19] investigated the effect of a mixture of shikonin/alkannin and their derivatives in RAW 264.7 macrophages stimulated with LPS. They concluded that this particular mixture inhibited LPS-induced nitric oxide and prostaglandin $\mathrm{E}_{2}$ production, iNOS protein expression, and ERK, I $k B \alpha$, and NF- $k B$ activation. In all cases, the effect of the shikonin/alkannin mixture was higher than that of the other derivatives.

In contrast to Staniforth et al. [17], who were unable to demonstrate that shikonin inhibited both ERK and NF- $k$ B activation in vivo, these two studies, carried out by two independent groups $[18,19]$, have demonstrated this inhibition both in vitro and in vivo. Since the administration of shikonin in vivo was topical in all of the studies, the discrepancies in the results are probably due to the different doses tested: Staniforth et al. [17] used shikonin at a dose of $100 \mu \mathrm{g} /$ site/mouse, while Andújar et al. [18] calculated an $\mathrm{ID}_{50}=1.0 \mathrm{mg}$ per ear.

Lu et al. [20] studied the effect of shikonin in two other classic mouse models widely used in research on drugs with anti-inflammatory activity: xylene-induced auricle swelling and acetic acid-induced capillary permeability increase. The authors concluded that shikonin efficiently inhibited auricle swelling in a dose-dependent manner, with $4 \mathrm{mg} / \mathrm{kg}$ of shikonin yielding the same effect as dexamethasone at $2.5 \mathrm{mg} / \mathrm{kg}$. In vitro, they demonstrated that shikonin inhibited TNF- $\alpha$ production in LPS-stimulated rat primary macrophages as well as NF- $k$ B translocation from the cytoplasm to the nucleus. Moreover, at $1 \mu \mathrm{M}$ shikonin blocked NF- $k$ B nuclear translocation via inhibition of proteasome-mediated $\mathrm{I} \kappa \mathrm{B} \alpha$ degradation and induced cell death by inhibiting the proteasome in macrophage cells. The principal targets studied for shikonin are summarized in $\bullet$ Fig. 2.

\section{Shikonin in autoimmune-mediated inflammatory diseases}

Shikonin in arthritis: In vivo, shikonin was found to be active in a mouse model of established collagen-induced arthritis, but not in the early arthritis [21]. Indeed, they observed that shikonin had no effect on the influx of inflammatory cells and tissue damage during the early arthritis. However, the intraperitoneal injection of shikonin ( $5 \mathrm{mg} / \mathrm{kg}$ ) for 10 days after arthritis had been established significantly improved the macroscopic appearance and decreased cartilage destruction, reducing the expression of the Th1 cytokines TNF- $\alpha$ and IL-12 in both the synovial tissue and the articular cartilage through a mechanism involving T-bet. In contrast, the Th2 cytokines IL-10 and IL-4 remained elevated throughout treatment due to an increase in GATA-binding protein-3 expression. The expression levels of IL-6 were also inhibited.

More recently, Kim et al. [22] published a study on the efficacy of shikonin in protecting the cartilage in a murine model of rheumatoid arthritis, also induced by collagen. In this case, the authors used a dose of $2 \mathrm{mg} / \mathrm{kg}$ of shikonin administered orally daily for 35 days. As in the previous study, the incidence, bone mineral density, bone mineral content, and joint histopathology all improved significantly, with a decrease in inflammation, joint destruction, and matrix metalloproteinase- 1 production and a concomitant increase in the tissue inhibitor of metalloproteinase-1.

These two studies suggest that shikonin may be a good candidate for the development of a cartilage protective medicine. The transcription factors GATA-3 and T-bet regulate the differentiation of Th cells into either Th1 cells, which are responsible for cell-mediated immune defense against intracellular pathogens, or Th2 cells, which defend the host against extracellular parasites [23]. This polarization of Th cells is important because Th1 cells are 
considered to play a central role in autoimmune diseases. Shikonin's ability to switch the established polarization by inhibiting T-bet and increasing GATA-3 expression could be crucial in the treatment of such diseases for which an effective, definitive therapy has yet to be developed.

Shikonin in inflammatory bowel disease: Inflammatory bowel disease encompasses several pathologies that share various clinical and pathophysiological characteristics with rheumatoid arthritis. As immune-mediated inflammatory diseases, they are all treated with similar disease-modifying drugs, such as methotrexate, sulfasalazine, or more recently anti-TNF agents [24]. In a recent study, Andújar et al. [25] determined the effect of shikonin (25 mg/kg, p.o. twice during the experiment) on a mouse model of acute ulcerative colitis induced in Balb/C mice by oral administration of $5 \%$ dextran sodium sulfate. The authors found that treatment with shikonin prevented the shortening of the colorectum and decreased weight loss by $5 \%$ while improving the appearance of feces and preventing bloody stools. Moreover, MPO activity was reduced as well as the expression of COX-2, the activation of NF- $k$ B and that of STAT3. Cytokine production (TNF- $\alpha$, IL-1 $\beta$, and IL-6) was also inhibited. Similar results were obtained in primary macrophage cultures. Although these authors did not evaluate the effect of shikonin on T-bet and GATA-3 expression, the inhibition of Th1 cytokines in this model indicates that a similar mechanism of action for shikonin is at work in both arthritis and colitis.

Shikonin in asthma: The usefulness of shikonin as an antiallergic agent has also been described. Tanako-Ohmuro et al. [26] reported that shikonin inhibits in a dose-dependent manner $\left(\mathrm{IC}_{50}=2.6 \mu \mathrm{M}\right)$ histamine release mediated by anti-immunoglobulin $\mathrm{E}$ antibodies in basophils isolated from the blood of healthy volunteers. As a target in the signal cascade of IgE-mediated activation, the authors proposed spleen tyrosine kinase. More recently, the effects of shikonin on a murine model of asthma and the maturation of dendritic cells derived from bone marrow have also been studied [27]. The results demonstrated not only that shikonin at $0.3 \mu \mathrm{M}$ is able to inhibit the in vitro maturation of dendritic cells but also that in vivo ( $4.5 \mu \mathrm{g}$, once daily for 5 days) it inhibits the allergic reaction and airway hyperresponsiveness in asthmatic mice. To gain insight into the mechanism of action of shikonin as an antiallergic drug, Lee et al. [28] studied the effects of shikonin on TPA plus cAMP-induced Th2 cytokine production in activated EL-4 murine T-lymphoma cells. These cells produce IL- 4 and IL- 5 but not interferon- $\gamma$ which is produced by Th2 cell-like cells and is responsible for allergic reactions. Shikonin $(0.3 \mu \mathrm{M})$ directly inhibited the Th2 responses in T cells by reducing the expression of IL-4, IL-5, and the transcription factors (GATA)-3 and c-Maf, but not T-bet. Moreover, shikonin suppressed the phosphorylation of $\mathrm{p} 38$, I $k \mathrm{~B}$ kinase $\beta$, and I $k \mathrm{~B} \alpha$ while inhibiting the subsequent $\mathrm{I} \kappa \mathrm{B} \alpha$ degradation induced by TPA + cAMP. However, it seemed to have no effect on the TPA + cAMPinduced activation of ERK and c-Jun $\mathrm{N}$-terminal kinase. These results appear to be contradictory with those obtained by Dai et al. [21], Kim et al. [22], and Andújar et al. [25]. However, while these authors published their results based on in vivo studies, Lee et al. [28] were working with a Th2-like cell line. Although the importance of in vitro studies in elucidating mechanisms of action is undeniable, it should be remembered that the complex immune regulatory networks that act throughout the whole organism might completely modify the expected outcome based on results obtained in vitro. Another possible explanation for the aforementioned discrepancies may be the different doses tested. In any case, more studies should be carried out to shed light on how different doses of shikonin may polarize the immune system towards a Th1 or a Th2 response.

Shikonin in lupus nephritis: Wang et al. [29] orally administered shikonin (24 and $40 \mathrm{mg} / \mathrm{kg} /$ day) to NZB/W F1 mice for 14 weeks and reported that proteinuria was dose-dependently reduced as well as circulating adhesion molecules. Moreover, they observed a downregulation in intercellular adhesion molecule- 1 and vascular cell adhesion molecule- 1 mRNA expression in the kidneys of the treated mice. The histological examination of the kidney also confirmed that renal glomerular lesions were alleviated after shikonin treatment. The results from this study reveal a possible therapeutic effect of shikonin on lupus nephritis through a mechanism which the authors hypothesize to be an inhibition of the cytokine-induced cellular adhesion molecules. This cytokine inhibition would be a common mechanism displayed in other autoimmune models in which shikonin has been studied, although Wang et al. [29] did not carry out any experiment to confirm this. Effect of shikonin on other animal models: Shikonin has recently also been studied in models of acute pancreatitis [30] and acute lung injury [31]. In the case of the murine model of cerulein-induced acute pancreatitis [30], shikonin $(50 \mathrm{mg} / \mathrm{kg})$ significantly reduced the pancreatic histological scores and the serum amylase and lipase activities. Moreover, the production of the cytokines TNF- $\alpha$, IL- $1 \beta$, and IL- 6 was reduced, as well as MPO activity. This cytokine and MPO inhibition was also accompanied by a reduced NF- $k$ B activity. Shikonin also decreased the productions of the proinflammatory cytokines TNF- $\alpha$ and IL- $1 \beta$, and the concentrations of MPO and NO in acute lung injury induced by LPS in mice in a model of acute lung injury [31]. The pretreatment of mice with shikonin significantly suppressed LPS-induced activation of COX-2 and iNOS, as well as the NF- $k$ B DNA-binding activity in lung tissues.

These molecular mechanisms described all coincide with those observed in the other mouse models reviewed. It seems reasonable, then, to conclude that the most feasible mechanism involved in shikonin's anti-inflammatory activity is the inhibition of the NF- $k$ B pathway, as demonstrated by the different in vivo and in vitro studies cited. The downregulation of this pathway explains perfectly the other effects observed, such as the inhibition of the production of cytokines, adhesion molecules, and other inflammatory mediators. In fact, using LPS-stimulated THP-1 monocytes, Chiu et al. [32] established that shikonin significantly inhibited the early expression of approximately 50 genes, some of them corresponding to relevant cytokines implicated in inflammation (TNF- $\alpha$, IL- $1 \beta$, IL-4), chemokines and inflammatory modulators. Their results led them to the conclusion that shikonin strongly inhibits macrophage activation (results which have been confirmed by other authors [18-20,25]), chemotaxis, and T-lymphocyte recruitment. In this same line, Li et al. [33] have recently confirmed that shikonin is able to suppress human T-cell proliferation, IL-2 and IFN- $\gamma$ secretion, CD69 and CD25 expression, as well as cell cycle arrest activated by costimulation of TPA/ionomycin or OKT-3/CD28 monoclonal antibodies. These effects are mediated by shikonin through the suppression of the NF- $k$ B signaling pathway via inhibition of the IKK $\alpha / \beta$ phosphorylation, I $k$ $\mathrm{B} \alpha$ phosphorylation and degradation, and NF- $k \mathrm{~B}$ nuclear translocation by directly decreasing IKK $\beta$ activity. Shikonin also suppressed JNK phosphorylation in the MAPKs pathway of T cells. The main animal models in which shikonin has been tested as well as the reported molecular targets for shikonin have been summarized in two tables ( $\bullet$ Tables 1 and 2 ). 
Table 1 Principal molecular targets implicated in inflammatory diseases that are inhibited by shikonin.

\begin{tabular}{|c|c|}
\hline \multicolumn{2}{|c|}{ Molecular targets of inflammation inhibited by shikonin } \\
\hline \multicolumn{2}{|c|}{ Cytokines } \\
\hline TNF $\alpha$ & {$[17,20,21,25,30-32]$} \\
\hline IL-12 & [21] \\
\hline IL-6 & {$[21,25,30,31]$} \\
\hline $\mathrm{IL}-1 \beta$ & {$[25,30-32]$} \\
\hline IL-2 & [33] \\
\hline IFNy & [33] \\
\hline \multicolumn{2}{|l|}{ MAPK } \\
\hline ERK1/2 & {$[18,19]$} \\
\hline JNK & [33] \\
\hline \multicolumn{2}{|l|}{ Pro-inflammatory enzymes } \\
\hline COX-2 & {$[18,25,31]$} \\
\hline $\mathrm{I} \kappa \mathrm{B} \alpha$ & {$[18-20,33]$} \\
\hline iNOS & {$[18,19,31]$} \\
\hline MMP-1 & {$[22]$} \\
\hline MPO & {$[25,30,31]$} \\
\hline Proteasome & {$[20]$} \\
\hline Serum amylase activity & [30] \\
\hline Serum lipase activity & [30] \\
\hline Spleen tyrosine kinase & {$[26]$} \\
\hline \multicolumn{2}{|l|}{ Transcription factors } \\
\hline $\mathrm{NF}-\kappa \mathrm{B}$ & {$[18-20,25,30,31,33]$} \\
\hline T-bet & {$[21,22]$} \\
\hline STAT3 & [25] \\
\hline \multicolumn{2}{|l|}{ Other mediators } \\
\hline NO & [30] \\
\hline Intracellular adhesion molecule & {$[29]$} \\
\hline $\mathrm{PGE}_{2}$ & [19] \\
\hline Vascular cell adhesion molecule & [29] \\
\hline
\end{tabular}

\section{Antioxidant Effects of Shikonin}

The antioxidant properties of shikonin and its derivatives were studied, and their structure-activity relationship was approximately established by Assimopoulou et al. [34]. All the hydroxynaphthoquinones studied by these authors showed high radical scavenging activity against the DPPH radical; therefore, they established that the presence of the naphthoquinone moiety is essential for the activity, while the side chain possibly plays a minor role. Moreover, in a recently published paper, Jin and Bai [35] studied the radical scavenging activity of shikonin and its derivatives by using density functional theory and demonstrated that the introduction of acyl groups decreases the ionization potential values compared with those of shikonin. However, Zeng [15] obtained a poor activity for shikonin as a DPPH radical scavenger, with an $\mathrm{IC}_{50}$ value of $56.3 \mu \mathrm{g} / \mathrm{mL}$, whereas the $\mathrm{IC}_{50}$ values against ABTS and $\mathrm{Fe}^{2+}$ ascorbate/rat brain were $1.93 \mu \mathrm{g} / \mathrm{mL}$ and $6.3 \mu \mathrm{g} /$ $\mathrm{mL}$, respectively. Moreover, shikonin had no activity in the superoxide and xanthine oxidase tests.

An application for shikonin's antioxidant properties has been suggested by Nishizawa et al. [36]: a possible use of shikonin for cigarette filters. These authors quantified the peroxyradicals recovered from cigarette smoke and compared the scavenging activity of shikonin vs. other antioxidants, such as ascorbic acid, reduced glutathione, and epigallocatechin gallate, concluding that, among all the antioxidants studied, shikonin alone exerted the best scavenging activity. Its effectiveness against peroxyradicals together with its heat resistance, non-volatility, and high affinity
Table 2 In vivo models used for the study of the anti-inflammatory activity of shikonin.

\begin{tabular}{ll} 
Anti-inflammatory effect of shikonin in vivo & \\
In vivo model & Reference \\
\hline TPA-induced mouse ear edema & {$[18]$} \\
Xylene-induced auricle swelling & {$[20]$} \\
\hline Acetic acid-induced capillary permeability increase & {$[20]$} \\
\hline Arthritis & {$[21,22]$} \\
\hline Inflammatory bowel disease & {$[25]$} \\
\hline Asthma & {$[27]$} \\
Lupus nephritis & {$[29]$} \\
\hline Acute pancreatitis & {$[30]$} \\
\hline Lung injury & {$[31]$}
\end{tabular}

to the filter are all characteristics that made the authors suggest the possible use of shikonin for cigarette filters.

\section{Neuroprotective Properties of Shikonin \\ $\nabla$}

Shikonin has also been shown to exhibit a neuroprotective effect against the damage caused by ischemia/reperfusion in adult male Kunming mice [37]. This finding implies that treatment with this molecule may exert a significant protective effect against cerebral brain injury. Shikonin administered at a dose of $50 \mathrm{mg} / \mathrm{kg}$ (i.g.) once a day for three days before middle cerebral artery occlusion and once again $2 \mathrm{~h}$ after the onset of ischemia resulted in a significant decrease in neurological deficit scores, infarct size, and levels of malondialdehyde, carbonyl, and reactive oxygen species. It also attenuated neuronal damage and the upregulation of superoxide dismutase, catalase, and glutathione peroxidase activities while reducing the glutathione/glutathione disulfide ratio. These results indicate that the neuroprotective effects of shikonin against cerebral ischemia/reperfusion injury may be due to its antioxidant effects.

This neuroprotective activity of shikonin has also been described by Nam et al. [38]. These authors examined the effect of shikonin and five of its derivatives (acetylshikonin, isobutyrylshikonin, isovalerylshikonin, $\beta, \beta$-dimethylacrylshikonin, and $\beta$-hydroxyisovalerylshikonin) on microglial cells which are the prime effectors in immune and inflammatory responses of the central nervous system. Their results indicate that two of shikonin's derivatives (isobutyryl- and isovalerylshikonin) were even more effective than shikonin in repressing microglial LPS-induced activation.

Finally, in a recently published paper, Esmaeilzadeh et al. [39] demonstrated that shikonin protected dopaminergic neurons against 6-hydroxydopamine-induced neurotoxicity. In fact, the treatment of PC12 cells (rat pheochromocytoma) with shikonin $(10 \mu \mathrm{M})$ protected nearly $70 \%$ of the cells against toxicity, via both glutathione-dependent and direct anti-apoptotic pathways, because shikonin avoided the depletion of intracellular glutathione and upregulated glutathione-independent superoxide dismutase mRNA. These results are in concordance with those of Wang et al. [37]. Moreover, shikonin upregulated Bcl-2, downregulated Bax and prevented cell nuclei from undergoing morphological changes typical of apoptosis. 


\section{Cardioprotective Effects of Shikonin}

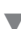

Two different studies have suggested a possible cardioprotective effect of shikonin that would be related to its anti-inflammatory and antioxidant effects. To this respect, Andrikopoulos et al. [40] reported that shikonin at $10 \mu \mathrm{M}$ gave a percentage of protection against the in vitro LDL oxidation of $61 \%$; however, it did not show a clear dose-dependency. Later, Yoshida et al. [41] did describe a dose-dependent inhibition of the rat thoracic aorta relaxation in response to acetylcholine ( $\mathrm{pD}_{2}$ value 6.29 ) and the LPS-induced NO production ( $82 \%$ at $1 \mu \mathrm{M}$ ) in RAW 264.7 cells. Because shikonin inhibits all isoforms of NOS ( $\left.\mathrm{IC}_{50} 4-7 \mu \mathrm{M}\right)$, it was hypothesized that inhibition of NOS is implied in both events. The anti-inflammatory studies carried out with shikonin all coincide in its ability to inhibit inducible NOS; therefore, it is reasonable to suspect, as Yoshida et al. [41] did, that shikonin could also have an effect on the endothelial NOS. However, in view of the apparent discrepancy of these two studies on the cardiovascular effect of shikonin, more studies should be carried out in this field in order to confirm this effect.

\section{Anticancer Activity of Shikonin}

$\nabla$

The anticancer effects of $L$. erythrorhizon have been demonstrated in vitro in B16F10 murine melanoma cells [42]. The $n$-hexane extract was found to inhibit the growth of melanoma cells with an $\mathrm{IC}_{50}$ of $2.73 \mu \mathrm{g} / \mathrm{mL}$. Moreover, L. erythrorhizon treatment at a dose of $10 \mathrm{mg} / \mathrm{kg}$ for 21 days in experimental mice implanted with tumors resulted in significant reductions in tumor growth (43\%) and weight (36\%). Five naphthoquinone compounds (shikonin, deoxyshikonin, $\beta$-hydroxyisovalerylshikonin, acetylshikonin, and isobutyrylshikonin) purified from the extract were described as being responsible for its anticancer activity. In a similar study, Kretschmer et al. [43] described the ability of a petroleum ether-soluble extract of the roots of Onosma paniculata (containing several shikonin derivatives, namely, $\beta$-hydroxyisovalerylshikonin, acetylshikonin, dimethylacrylshikonin, and a mixture of $\alpha$ methylbutyrylshikonin and isovalerylshikonin) to exert strong cytotoxicity against eight cancer cell lines and MRC-5 lung fibroblasts. However, the isolated shikonin molecule has been more extensively studied than the root extract as an anticancer agent. A wide spectrum of anticancer mechanisms of action have been described for shikonin: suppression of NF- $k$ B-regulated gene products [44], inhibition of proteasome activity [45], ROS generation [46], inhibition of tumor-specific pyruvate kinase-M2 [47, 48], cell cycle arrest [49], or induction of necroptosis [50], among others. Since a review on the anticancer activity of shikonin has recently been published [51], the present review will only focus on the very recently published papers, therefore being an update of the previously published paper. In this sense, Huang et al. [52] have only recently published their results on shikonin's ability (at a dose of $3 \mu \mathrm{M}$ ) to induce cell death in C6 and U87 glioma cells through a mechanism that involved the induction of necroptosis, with the participation of the RIP-1 pathway and an increase in oxidative stress. In this same line, Piao et al. [53] tried to examine in more depth the molecular mechanisms involved in cell death initiation by shikonin, both in caspase-dependent and caspaseindependent cell death. In their study they demonstrate that shikonin at $1 \mu \mathrm{M}$ induced caspase-dependent apoptosis in U937 cells after $6 \mathrm{~h}$ with an increase in DNA fragmentation, intracellular ROS, low mitochondrial membrane potential, and with the ex- pression of Noxa and tBid proapoptotic proteins. Necroptosis was also detected but with a higher dose of shikonin $(10 \mu \mathrm{M})$. At $10 \mu \mathrm{M}$, shikonin induced a greater release of cytochrome $\mathrm{c}$ from the mitochondria and of lactate dehydrogenase, but Noxa and tBid were not affected. Interestingly, exogenous glutathione completely inhibited $1 \mu \mathrm{M}$ apoptosis and converted $10 \mu \mathrm{M}$ necroptosis to apoptosis. In other words, shikonin depletes glutathione dose-dependently, and therefore the oxidative stress induced by shikonin plays an important role in cell death initiation. Moreover, these authors analyzed the gene expression during these processes and found that 353 genes were significantly regulated by $1 \mu \mathrm{M}$ shikonin (that is, during apoptosis) and 85 genes by $10 \mu \mathrm{M}$ shikonin treatment (that is, in necroptosis induction). Among these genes, the transcription factor 3 and DNA-damage-inducible transcript 3 were highly expressed at shikonin-induced apoptosis $(1 \mu \mathrm{M})$, while TNF expression mainly increased at $10 \mu \mathrm{M}$ treatment.

In addition, Ahn et al. [54] also confirmed the ROS-mediated induction of apoptosis in several cancer cell lines (cervical cancer cell line HeLa, colon cancer cell line Hct116, hepatocellular carcinoma cell line Hep3B, and a lung cancer cell line A549). Their results indicate that shikonin exerts a cytotoxic effect in these cell lines, having a 50\% growth inhibition range of 0.5-3.0 $\mu \mathrm{M}$. Moreover, they demonstrated that elevated ROS levels due to prolonged exposure to shikonin activated ASK1 and p38 MAPK and downregulated $\mathrm{p} 21^{\mathrm{Cip} 1}$, which results in $\mathrm{G} 1$ arrest, all these events leading to the promotion of apoptosis. These two studies $[53,54]$ give a deeper insight into the possible mechanism of action by which shikonin induces cell death through a pro-oxidant mechanism. Zhang et al. [55] also justify some properties of shikonin on the basis of its pro-oxidative effects. In this sense, working with HL-60 cells, they demonstrated that treatment with shikonin reduced the glutathione/glutathione disulfide ratio, and the nonenzymatic antioxidant, 4,5-dihydroxybenzene-1,3-disulfonate, showed a remarkable recovery effect on the shikonin-induced decrease in glutathione. Also, when the enzymatic antioxidant and ROS scavengers, superoxide dismutase and catalase were added to cell cultures prior to shikonin, a similar trend was observed in the suppression of ROS production but also in the restoration of glutathione. Based on these results, the authors conclude that shikonin has a specific role of redox homeostasis in regulating HL-60 cell differentiation.

A very recently published paper describes the antimetastasis properties of shikonin [56]. The authors treated non-small cell lung cancer A549 cells with shikonin $8 \mu \mathrm{M}$ for $24 \mathrm{~h}$ and demonstrated that shikonin significantly inhibited cell proliferation. When cells were treated with a dose inferior to $2.0 \mu \mathrm{M}$ for $24 \mathrm{~h}$, cell adhesion to the extracellular matrix was significantly suppressed, as well as invasion and migration.

Moreover, they showed that the mechanisms by which shikonin was able to exert this antimetastasis effect involved a reduction in the expression of integrin $\beta 1$ at the mRNA and protein levels and a repression of the phosphorylation of ERK1/2.

These results, together with others previously reviewed [51], demonstrate that shikonin is able to inhibit or modulate a wide spectrum of cellular targets associated with cancer. Since multiple mechanisms are involved in the development of cancer, a molecule such as shikonin seems to be a promising anticancer agent. 


\section{Antimicrobial Activity}

$\nabla$

\section{Antibacterial activity}

In general, shikonin and its derivatives have been shown to be active against gram-positive bacteria such as Staphylococcus aureus, Enterococcus faecium, and Bacillus subtilis at MICs ranging from 0.30 to $6.25 \mathrm{mg} / \mathrm{mL}$, as well as against various species of lactic acid bacteria. In contrast, they are inactive against gram-negative bacteria such as Escherichia coli, Pseudomonas aeruginosa, and Micrococcus luteus [2,57], although a study published in 2011 by Ding et al. [58] demonstrated that at a dose of $200 \mu \mathrm{M}$, shikonin inhibits biofilm formation by P. aeruginosa and Stenotrophomonas maltophilia. Initially it was thought that shikonin's antibacterial activity derived from a bacteriostatic effect, but a kinetic study revealed that the compound possesses bactericidal properties [2]. In the same study, it was suggested that shikonin could be a useful therapeutic agent against methicillin-resistant S. aureus. In 2002, Shen et al. [59] analyzed the activity of shikonin and some derivatives against methicillin-resistant $S$. aureus and vancomycin-resistant E. faecium and E. faecalis. In this test, shikonin showed an MIC of $6.25 \mu \mathrm{g} / \mathrm{mL}$ against $S$. aureus (both methicillinresistant and not resistant), and 50 and $25 \mu \mathrm{g} / \mathrm{mL}$ against $E$. faecicum and E. faecalis, respectively. This study corroborates shikonin's inhibitory activity against $S$. aureus, however, the ester derivatives showed greater potency, especially its methylbutyryl derivative which had MIC values of $1.56 \mu \mathrm{g} / \mathrm{mL}$ against the three resistant microorganisms tested. Recently, Li et al. [60] published a paper in which they recorded an MIC value of $125 \mu \mathrm{g} / \mathrm{mL}$ against $S$. aureus, but these authors did not study the effect of shikonin on the methicillin-resistant strain. The MIC difference between both studies is remarkable: Li et al.'s [60] results are 20 times higher than Shen et al.'s [59], therefore more studies to confirm which is the correct MIC should be carried out. In any case, it is worth mentioning that the two authors used different approaches to determine the MIC.

In 2004, Kuo et al. [61] demonstrated that shikonin inhibits the growth of Helicobacter pylori in a dose-dependent manner $\left(\mathrm{IC}_{50}=18 \mu \mathrm{M}\right)$. Some enzymes of enteric bacteria, including Klebsiella pneumoniae, Salmonella group B, S. aureus, E. coli, and H. pylori, are known to exhibit NAT activity, which contributes to the metabolic activation of chemical carcinogens. In the aforementioned study, the authors were also able to demonstrate the ability of shikonin $(4 \mu \mathrm{M})$ to inhibit NAT-mediated $N$-acetylation.

\section{Antifungal activity}

There is less consensus in the literature with regard to the antifungal properties of shikonin, alkannin, and their derivatives. As recorded by Papageorgiou et al. [2], the antidermatophytic properties of shikonin and deoxyshikonin were tested against five fungi: Saccharomyces sake, Trichophyton rubrum, T. mentagrophytes, T. tonsulans var. sulfureum, Microsporum gypseum, and Epidermophyton fluccosum (MIC $<25 \mathrm{mg} / \mathrm{mL}$ ). Both were found to exert a fungistatic rather than fungicidal action, with deoxyshikonin generally being the more active compound. An ointment containing deoxyshikonin as the main component to control athlete's foot was patented, along with other antimicrobial preparations containing mainly shikonin. Papageorgiou et al. [2] reviewed conflicting studies regarding the activity of shikonin and deoxyshikonin against Candida albicans; while several researchers found shikonin and its derivatives to be inactive against C. albicans, others claimed it was active. In 2002, a paper published by Sasaki et al. [62] comparing the effects of shikonin and the standard antifungal fluconazole showed that the fungicidal activity of shikonin ( $\mathrm{MIC}=4 \mathrm{mg} / \mathrm{mL}$ ) was 4 times higher against Candida krusei, 2 times higher against Saccharomyces cerevisiae, and the same as that of fluconazole against $C$. glabrata. Deoxyshikonin also exhibited a more potent activity against $C$. krusei and $S$. cerevisiae than fluconazole. While other derivatives tested proved to be less active than the standard, all showed some activity. More recently, Miao et al. [63] demonstrated that shikonin is active against $C$. albicans, including several fluconazole-resistant strains, against which shikonin $\left(\mathrm{MIC}_{80}=4 \mu \mathrm{g} / \mathrm{mL}\right.$ ) was shown to be $>16$ times more potent than the standard $\left(\mathrm{MIC}_{80}>64 \mu \mathrm{g} / \mathrm{mL}\right.$ ). Moreover, these same authors found that treatment with shikonin increased the generation of endogenous reactive oxygen species while decreasing mitochondrial membrane potential. They also demonstrated that antioxidants such as $\mathrm{N}$-acetylcysteine and glutathione reduced the antifungal activity of shikonin against C. albicans. Their analyses identified 9 differentially expressed genes related to glycolysis-related genes (CDC19 and $H X K 2$ ), fermentation-related genes ( $A L D 5$ and $A D H 1$ ), antioxidant defense-related genes (SOD2 and SOD5), a thioredoxin reductaserelated gene (TRR1), a mitochondrial respiratory electron transport chain-related gene (MRF1), and a reduced NADPH oxidoreductase-related gene (EBP1). These results suggest that mitochondrial aerobic respiration shifts and increases in endogenous reactive oxygen species may contribute to the action of shikonin against $C$. albicans.

This antifungal activity has many potential clinical applications, especially when combined with shikonin's anti-inflammatory and antibacterial activity, which may be useful to treat mouth ulcers and oral candidiasis resulting from the use of cytotoxic therapies and immunosuppressants, human immunodeficiency virus (HIV) infection, or opportunistic fungal infections in individuals in whom the use of corticosteroids is contraindicated.

\section{Antiparasitic activity}

Michaelakis et al. [64] evaluated the concentration-dependent effects of three pure synthetic natural products (alkannin, shikonin, and shikalkin) and three acetylated derivatives of shikonin against Culex pipiens (Culicidae: Diptera). As the authors note, previous studies had found that both shikonin and shikonin angelate exhibited a considerable larvicidal effect against Aedes aegypti larvae, with shikonin giving an $\mathrm{LC}_{50}$ value of $7 \mu \mathrm{g} / \mu \mathrm{L}$ while that of shikonin angelate was about $14 \mu \mathrm{g} / \mu \mathrm{L}$. Larvicidal bioassays revealed that $100 \%$ mortality was achieved with $10 \mu \mathrm{g} / \mu \mathrm{L}$ of shikonin, whereas higher doses were needed for other naphtoquinones: $30 \mu \mathrm{g} / \mu \mathrm{L}$ for alkannin, $20 \mu \mathrm{g} / \mu \mathrm{L}$ for shikalkin. The assays showed that a relatively high mortality rate (approximately $17 \%$ ) could be reached with shikonin concentrations as low as $2 \mu \mathrm{g} / \mu \mathrm{L}$. Naphthoquinones showed a higher larvicidal effect than other naturally-occurring biocides such as thymol and carvacrol, which have $\mathrm{LD}_{50}$ values of $36 \mu \mathrm{g} / \mu \mathrm{L}$ and $37.6 \mu \mathrm{g} / \mu \mathrm{L}$, respectively. In this same study, the authors synthesized three acetylated derivatives of shikonin in order to elucidate the structure-activity relationships of shikonin-type compounds with larvicidal activity against $C$. pipiens. Their results showed that although hydroxylic groups seem to play a secondary role in the efficacy of these compounds, the quinone moiety is essential. In this context, Ali et al. [65] demonstrated that shikonin exhibits the highest toxicity for intracellular persisting Leishmania major GFP parasites, with an $\mathrm{IC}_{50}$ value of $1.9 \mu \mathrm{M}$. Moreover, when comparing shikonin with its parent analogue, naphtazarin, they concluded that the presence of the 1'-hydroxy-3'-isohexenyl substituent at C-3 en- 
hanced the activity and that esterification of the side chain hydroxyl reduced the antileishmanial activity. The presence of the free hydroxyl group is apparently a major contributing factor in shikonin's antileishmanial activities.

\section{Antiviral activity}

A recent upsurge of studies on the antiviral activity of shikonin have also produced promising results, with shikonin reportedly exhibiting in vitro activity against HIV type 1 [66,67]. In particular, Chen et al. [66] examined the effects of shikonin against the HIV-1 receptor antagonist activity of multiple chemokines. At nM concentrations, shikonin inhibited monocyte chemotaxis and calcium flux in response to a variety of chemokines. It downregulated surface expression and mRNA expression of CCR5, a primary HIV-1 co-receptor, in macrophages to a greater degree than the other receptors. Additionally, shikonin inhibited the replication of both a multidrug-resistant strain and pediatric clinical isolates of HIV in human peripheral blood mononuclear cells, with $\mathrm{IC}_{50}$ values ranging from 96 to $366 \mathrm{nM}$. Shikonin also effectively inhibited the replication of the HIV Ba-L isolate in monocytes/macrophages $\left(\mathrm{IC}_{50}=470 \mathrm{nM}\right)$. These results suggest that both the anti-HIV and the anti-inflammatory activities of shikonin may be related to its interference with chemokine receptor expression and function. The authors therefore consider shikonin, a naturally occurring, low-molecular-weight pan-chemokine receptor inhibitor, to be a potential lead molecule for the design and development of therapeutic agents against HIV

In 2011, Gao et al. [68] reported on the antiviral effects of shikonin against AdV3. These authors found that shikonin inhibited growth of AdV3 in a concentration-dependent manner (0.0156$1 \mu \mathrm{M})$, with a virus inhibition rate of $24-69 \%$. Specifically, expression of the hexon protein in AdV3 was higher in the virus control group than in the shikonin-treated groups, while the rate of shikonin-treated HeLa cell apoptosis decreased significantly with increasing concentrations of the drug. These results indicate that shikonin possesses anti-AdV3 capabilities and that the potential antiviral mechanism most likely involves the inhibition of both apoptosis and hexon protein expression of AdV.

More recently, several phytochemicals from Arnebia euchroma, including shikonin, have been assessed for their activity against the HCV [60]. Among the compounds tested, shikonin exhibited the most potent anti-HCV activity, with an effective concentration (EC) 50 value of $25 \mathrm{ng} / \mathrm{mL}$, far lower than that of the positive control ribavirin $\left(\mathrm{EC}_{50}=2.6 \mu \mathrm{g} / \mathrm{mL}\right)$, and $\mathrm{CC}_{50}$ of $1.1 \mu \mathrm{g} / \mathrm{mL}$. Shikonin thus seems to be an HCV replication inhibitor and may therefore be a good candidate for further optimization and drug development.

\section{Shikonin in Wound Healing \\ $\nabla$}

Medicinal use of the root of Lithospermum erythrorhizon dates back to the second century, but the first written reference to its therapeutic qualities can be found in a traditional Chinese medical text from 1596, Pen Ts'ao Kang Mu [4]. This medical compendium recommends using the root for the treatment of burns, anal ulcers, hemorrhoids, and skin wounds. It was not until 1976 that this property of shikonin was confirmed experimentally by Papageorgiou et al. [4]. Subsequent experimental animal models and clinical studies have demonstrated the efficacy of shikonin and alkannin esters in wound healing, leading to the appearance of a number of pharmaceutical formulations: Histoplastin $\operatorname{Red}^{\circledR}$
(Chropi), Epouloderm ${ }^{\circledR}$ (Farmalex), and Helixderm ${ }^{\circledR}$ (PNG Gerolymatos). Histoplastin $\operatorname{Red}^{\circledR}$ was tested in patients with chronic leg ulcers that had resisted previous attempts at treatment. After 5 to 6 weeks of application, complete healing, or at least a considerable reduction of the ulcer, was observed. The success rate was $80 \%$ with no inflammatory processes recorded during treatment [4]. Later studies confirmed the efficacy of this ointment for the treatment of decubitus ulcers, leprosy, anal fissures, and burns, against which, according to the authors, it was more effective than Betadine ${ }^{\circledR}$ (povidone-iodine) and Fucidine ${ }^{\circledR}$ (fusidic acid) [4].

In a recent study conducted by Karayannopoulou et al. [69], the effect of an alkannin/shikonin-based ointment on second intention wound healing of surgically created, acute, non-contaminated wounds in dog skin was compared with that of LRS. The histological findings point to the ability of the ointment to promote angiogenesis, collagen production, and epithelialization of the lesions, although the time to complete wound closure was not significantly higher than in the control group (which was given only LRS). These results contradict those of previous human clinical trials, which had demonstrated the compound's efficacy in clinical cases of chronic, severe, or contaminated wounds. The authors explained this difference by arguing that LRS is a lavage solution capable of achieving closure of an open wound once it is clean; however, in contaminated wounds, the use of antiseptics or antibiotics in the lavage solution may damage tissues. Therefore, in the case of contaminated wounds, the use of an alkannin/shikonin ointment may be more beneficial.

Hsiao et al. [70] recently generated a biochemical and proteomic platform to establish the molecular basis of the wound healing process induced by L. erythrorhizon and shikonin. In their study they found that both the ethanol extracts of L. erythrorhizon and shikonin $(100 \mathrm{nM})$ were able to promote cell proliferation in fibroblasts by up to $25 \%$ and decrease cell migration. The proteomic analysis identified twenty-two differentially expressed proteins when fibroblast cells were treated with the extract or with shikonin; these proteins were categorized into six hierarchical clusters entailing antioxidant activity, apoptosis, cell mobility, proliferation, metabolism of abnormal proteins, and collagen secretion. This indicates that the efficacy of $L$. erythrorhizon in wound healing may derive from a synergistic effect on a number of factors. Assays by these same authors using an animal model confirmed that the ethanol extract of $L$. erythrorhizon was able to accelerate wound healing on the back flank of SD rats.

More recently, Andújar et al. [71] demonstrated that shikonin $(1 \mu \mathrm{M})$ promoted wound healing in intestinal epithelial cells (IEC-18) through a mechanism that involved the increase of cell migration and $\operatorname{TGF} \beta_{1}$ induction, without increasing cell proliferation. However, the apparently contradictory results to those published by Hsiao et al. [70] could be explained by the fact that the cell lines studied are different, and, although both fibroblasts and IEC are implicated in wound healing processes, they have different roles. In any case, the wound healing effects of the root of L. erythrorhizon have been known for centuries in traditional Chinese medicine; therefore, although the data published today may seem contradictory, a deeper study of this property is worth doing in order to elucidate the mechanism of action involved in this demonstrated activity of shikonin. 


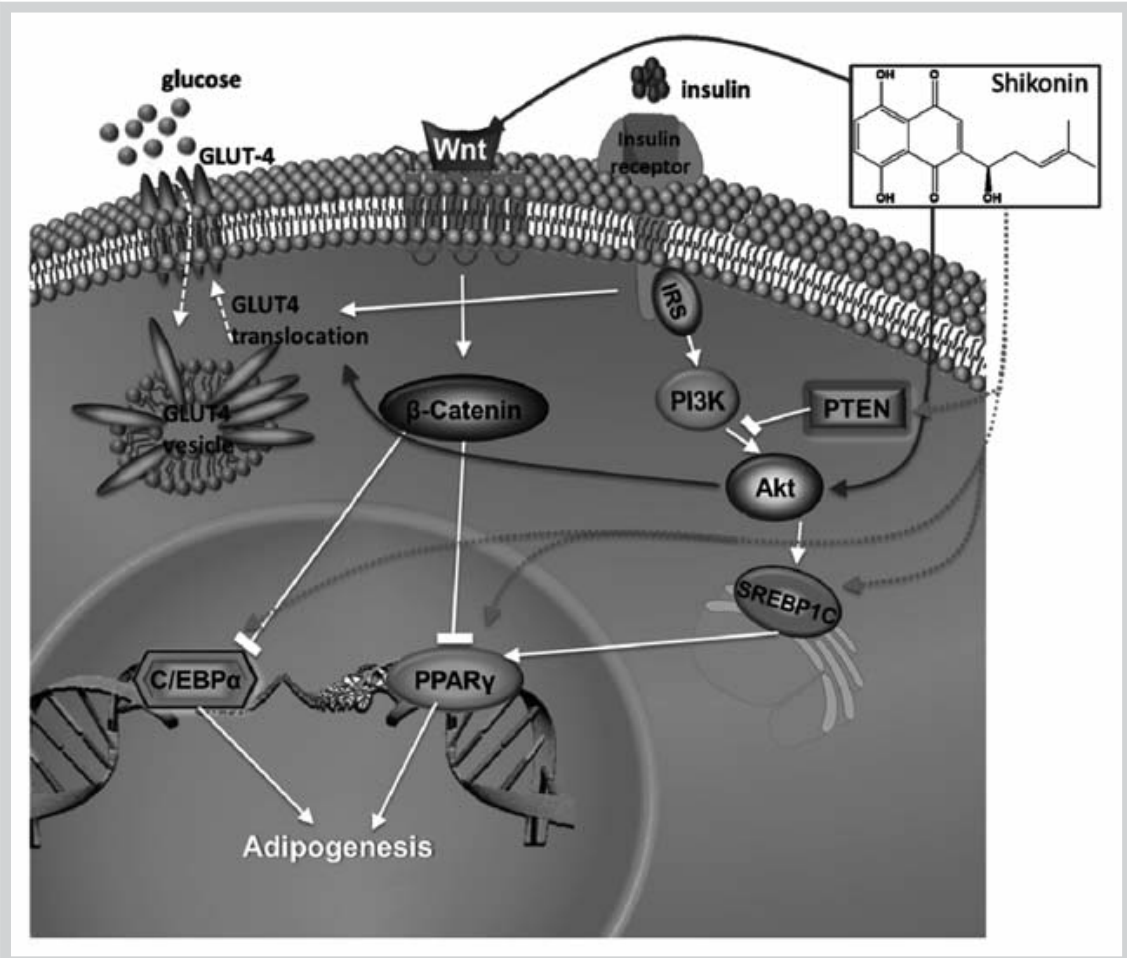

Fig. 3 Suggested targets for shikonin in glucose metabolism. C/EBP $\alpha$ (CCAAT/enhancer binding protein $\alpha$ ); GLUT4 (glucose transporter type 4); IRS (insulin receptor substrate protein); PI3K (phosphatidylinositol 3-kinase); PPARY (peroxisome proliferator-activated receptor $\gamma$ ); PTEN (phosphatase and tensin homolog); SREBP1C (sterol regulatory element-binding protein 1C). Black continuous line means activated by shikonin; dotted line means inhibited by shikonin.

\section{Shikonin and Glucose Metabolism}

$\nabla$

Recently, Gwon et al. [72] demonstrated the antiobesity effects of L. erythrorhizon on C57BL/6J mice fed with a high-fat diet and justified these effects by a mechanism mediated by the suppression of lipogenic genes in the liver and adipogenic transcription factors in white adipose tissue. They also established that acetylshikonin is, at least in part, responsible for these effects, but they did not study other derivatives present in the active extract, such as shikonin.

Other authors who have studied the antiobesity effect of shikonin have tried to elucidate its mechanism of action with regard to the adipogenesis of 3 T3-L1 cells [73]. Shikonin $\left(\mathrm{IC}_{50}=1.1 \mu \mathrm{M}\right)$ was found to inhibit fat droplet formation and triglyceride accumulation in 3 T3-L1 adipocytes through a mechanism involving the inhibition of genes implicated in lipid metabolism, such as FABP4 and LPL. Shikonin also inhibited the ability of PPAR $\gamma$ and C/EBP $\alpha$, the major transcription factors of adipogenesis, to bind to their target DNA sequences. Among the upstream regulators of adipogenesis, only SREBP1C was found to be downregulated by shikonin, which suggests that shikonin first inhibits the expression of SREBP1C and subsequently the expression of PPAR $\gamma$ and C/EBP $\alpha$, resulting in the suppression of lipid metabolizing enzymes and the reduction of fat accumulation. In a more recent paper [74], these same authors demonstrated that shikonin inhibits adipogenesis in vitro through the activation of the WNT/ $\beta$-catenin pathway to maintain the nuclear levels of $\beta$-catenin, thereby suppressing the expression of PPAR $\gamma, \mathrm{C} / \mathrm{EBP} \alpha$, and intracellular fat accumulation. The suggested targets for shikonin in glucose metabolism are compiled in 0 Fig. 3.

Kamei et al. [75] reported that treatment with $10-50 \mu \mathrm{M}$ of shikonin stimulated glucose uptake in 3 T3-L1 cells, not through the insulin receptor, insulin receptor substrate proteins, or phosphatidylinositol 3-kinase, but through an unidentified tyrosine kinase-mediated mechanism, resulting in the activation of Akt ki- nase and the stimulation of glucose uptake by GLUT4 translocation. Moreover, in Chinese hamster ovary cells transfected with the insulin receptor, shikonin $(30 \mu \mathrm{M})$ inhibited phosphatase and the tensin homolog, which may explain some of its insulinlike actions, especially increased glucose uptake [76]. Comparing the results obtained by Kamei et al. [75] and Nigorikawa et al. [76] to those published by Lee et al. [73,74], at least two possible targets for shikonin seem likely: (i) a target for the activation of glucose uptake and (ii) a target for the inhibition of fat accumulation, with the latter being efficiently inhibited at much lower concentrations.

In contrast to these findings, Öberg et al. [77] recently demonstrated that shikonin $(1 \mu \mathrm{M})$ increased glucose uptake in L6 myotubes to the same extent as insulin without affecting Akt-phosphorylation. These results seem to indicate that shikonin increases glucose uptake and, as a consequence, the intracellular levels of calcium in myotubes through an insulin-independent pathway. These authors concluded that shikonin enhances GLUT4-translocation from intracellular vesicles to the cell surface in L6 myoblasts, which is the same end mechanism as that used by insulin, but with a different intracellular signal (Akt-phosphorylation). For this reason, the authors hypothesize that shikonin may also affect glucose uptake in insulin-insensitive muscles. In addition, Öberg et al. [77] also demonstrated the beneficial effect of shikonin in vivo on spontaneously diabetic Goto-Kakizaki rats. When the rats were treated with $10 \mathrm{mg} / \mathrm{kg}$ of shikonin (i.p., once daily for 4 days), their levels of glucose in plasma decreased significantly. Moreover, an insulin sensitivity test $(0.5 \mathrm{U} / \mathrm{kg}$ insulin, s.c.) found significantly lower glucose levels in the shikonintreated group. The authors concluded that shikonin has a potentially beneficial effect on glucose metabolism, which makes it of considerable interest for developing novel treatments against type 2 diabetes. 


\section{Conclusion}

Modern science has gone a long way in demonstrating that the medicinal properties attributed by ancient folk traditions to the roots of $L$. erythrorhizon and other species of the Boraginaceae family have a scientific basis, lending credence to the use of these medicinal plants as a valuable source of modern drugs. In this sense, shikonin is being highlighted as a promising molecule due to all the beneficial effects that have been associated with its use. In fact, a molecule that is able to act simultaneously on various targets represents a great advantage in the treatment of different diseases. For example, the antimicrobial properties of shikonin are an added benefit to its wound healing properties since a drug that can both close a wound while preventing infection and swelling is highly beneficial. This example can be extended to the case of an inflammatory pathology such as ulcerative colitis, in which the anti-inflammatory activity of shikonin can clearly improve the evolution of the disease. Moreover, this particular type of disorder produces defects in the intestinal epithelial barrier, which in turn lead to an increase in the concentration of anaerobic bacteria with $N$-acetyltransferase activity, which can activate certain carcinogens. While shikonin exerts no antimicrobial activity against these bacteria, it is able to inhibit the enzyme and therefore play a protective role. Moreover, the wound-healing effects of this molecule also contribute to the treatment, since the sooner the intestinal ulcers heal, the sooner the patient recovers. There are even economic benefits when one drug has the same effect as three. Still, uncertainties remain, since shikonin shows different effects depending on the dose tested. Future studies should thus be developed to clarify and define not only the molecular targets for shikonin and some of its derivatives, but also the clinical potential of these molecules.

\section{Acknowledgements}

The authors thank the Spanish government (MICIIN and FEDER, grant SAF2009-10059-C03-01) for financial support. I. A. thanks the Generalitat Valenciana for her fellowship (grant BFPI/2008/ 040).

\section{Conflict of Interest}

$\nabla$

The authors state no conflict of interest.

\section{References}

1 Chen X, Yang L, Oppenheim JJ, Howard OMZ. Cellular pharmacology studies of shikonin derivatives. Phytother Res 2002; 16: 199-209

2 Papageorgiou VP, Assimopoulou AN, Couladouros EA, Hepworth D, Nicolaou KC. The chemistry and biology of alkannin, shikonin, and related naphthazarin natural products. Angew Chem Int Ed Engl 1999; 38: 270-301

3 Albreht A, Vovk I, Simonovska B. Addition of $\beta$-lactoglobulin produces water-soluble shikonin. J Agric Food Chem 2012; 60: 10834-10843

4 Papageorgiou VP, Assimopoulou AN, Ballis AC. Alkannins and shikonins: a new class of wound healing agents. Curr Med Chem 2008; 15: 32483267

5 Assimopoulou AN, Papageorgiou VP. Encapsulation of isohexenylnaphthazarins in cyclodextrins. Biomed Chromatogr 2004; 18: 240-247

6 Chen CY, Chen FA, Wu AB, Hsu HC, Kang JJ, Cheng HW. Effect of hydroxypropyl- $\beta$-cyclodextrin on the solubility, photostability and in vitro permeability of alkannin/shikonin enantiomers. Int J Pharm 1996; 141: $171-178$
7 Xia H, Tang C, Gui H, Wang X, Qi J, Wang X, Yang Y. Preparation, cellular uptake and angiogenic suppression of shikonin-containing liposomes in vitro and in vivo. Biosci Rep 2013; 33: e00020

8 Assimopoulou AN, Papageorgiou VP, Kiparissides C. Synthesis and release studies of shikonin-containing microcapsules prepared by the solvent evaporation method. J Microencapsul 2003; 20: 581-596

9 Huang YI, Cheng YH, Yu CC, Tsai TR, Cham TM. Microencapsulation of extract containing shikonin using gelatin-acacia coacervation method: a formaldehyde-free approach. Colloids Surf B Biointerfaces 2007; 58: 290-297

10 Han J, Chen TX, Branford-White CJ, Zhu LM. Electrospun shikoninloaded PCL/PTMC composite fiber mats with potential biomedical applications. Int J Pharm 2009; 382: 215-221

11 Kontogiannopoulos KN, Assimopoulou AN, Tsivintzelis I, Panayiotou C, Papageorgiou VP. Electrospun fiber mats containing shikonin and derivatives with potential biomedical applications. Int J Pharm 2011; 409: 216-228

12 Kontogiannopoulos KN, Assimopoulou AN, Hatziantoniou S, Karatasos K, Demetzos C, Papageorgiou VP. Chimeric advanced drug delivery nano systems (chi-aDDnSs) for shikonin combining dendritic and liposomal technology. Int J Pharm 2012; 422: 381-389

13 Kourounakis AP, Assimopoulou AN, Papageorgiou VP, Gavalas A, Kourounakis PN. Alkannin and shikonin: effect on free radical processes and on inflammation - a preliminary pharmacochemical investigation. Arch Pharm (Weinheim) 2002; 335: 262-266

14 Landa P, Kutil Z, Temml V, Vuorinen A, Malik J, Dvorkova M, Marsik P, Konoska L, Pribylova M, Schuster D, Vanek T. Redox and non-redox mechanism of in vitro cyclooxygenase inhibition by natural quinones. Planta Med 2012; 78: 326-333

15 Zeng YQ. Identificación y actividad farmacológica de principios de especies antiinflamatorias [PhD Thesis]. Valencia: University of Valencia; 2006

16 Kundakovic T, Fokialakis N, Dobric S, Pratsinis H, Kletsas D, Kovacevic N, Chinou I. Evaluation of the anti-inflammatory and cytotoxic activities of naphthazarine derivatives from Onosma leptantha. Phytomedicine 2006; 13: 290-294

17 Staniforth V, Wang SY, Shyur LF, Yang NS. Shikonins, phytocompounds from Lithospermum erythrorhizon, inhibit the transcriptional activation of human tumor necrosis factor $\alpha$ promoter in vivo. J Biol Chem 2004; 279: 5877-5885

18 Andújar I, Recio MC, Bacelli T, Giner RM, Ríos JL. Shikonin reduces oedema induced by phorbol ester by interfering with $\mathrm{I} \kappa \mathrm{B} \alpha$ degradation thus inhibiting translocation of NF- $k$ B to the nucleus. Br J Pharm 2010; 160: 376-388

19 Cheng YW, Chang CY, Lin KL, Hu CM, Lin CH, Kang JJ. Shikonin derivatives inhibited LPS-induced NOS in RAW 264.7 cells via downregulation of MAPK/NF- $k$ B signaling. J Ethnopharmacol 2008; 120: 264-271

20 Lu L, Qin A, Huang H, Zhou P, Zhang C, Liu N, Li S, Wen G, Zhang C, Dong $W$, Wang $X$, Dou QP, Liu J. Shikonin extracted from medicinal Chinese herbs exerts anti-inflammatory effect via proteasome inhibition. Eur J Pharmacol 2011; 658: 242-247

21 Dai Q Fang J, Zhang FS. Dual role of shikonin in early and late stages of collagen type II arthritis. Mol Biol Rep 2009; 36: 1597-1604

22 Kim YO, Hong SJ, Yim SV. The efficacy of shikonin on cartilage protection in a mouse model of rheumatoid arthritis. Korean J Physiol Pharmacol 2010; 14: 199-204

23 Chakir H, Wang H, Lefebvre DE, Webb J, Scott FW. T-bet/GATA-3 ratio as a measure of the Th1/Th2 cytokine profile in mixed cell populations: predominant role of GATA-3. J Immunol Methods 2003; 278: 157-169

24 Lesuis $N$, Befrits $R$, Nyberg $F$, van Vollenhoven RF. Gender and the treatment of immune-mediated chronic inflammatory diseases: rheumatoid arthritis, inflammatory bowel disease and psoriasis: an observational study. BMC Med 2012; 10: 82

25 Andújar I, Ríos JL, Giner RM, Cerdá-Nicolás M, Recio MC. Beneficial effect of shikonin on experimental colitis induced by dextran sulfate sodium in BALB/c mice. Evid Based Complement Alternat Med 2012; DOI: $10.1155 / 2012 / 271606$

26 Takano-Ohmuro H, Yoshida LS, Yuda Y, Morioka K, Kitani S. Shikonin inhibits IgE-mediated histamine release by human basophils and Sky kinase activity. Inflamm Res 2008; 57: 484-488

27 Lee CC, Wang CN, Lai YT, Kang JJ, Liao JW, Chiang BL, Chen HC, Cheng YW. Shikonin inhibits maturation of bone marrow-derived dendritic cells and suppresses allergic airway inflammation in a murine model of asthma. Br J Pharmacol 2010; 161: 1496-1511 
28 Lee CC, Kang JJ, Chiang BL, Wang CN, Cheng YW. Shikonin inhibited mitogen-activated IL-4 and IL-5 production on EL-4 cells through downregulation of GATA-3 and c-Maf induction. Life Sci 2011; 89: 364-370

29 Wang XC, Feng J, Huang F, Fan YS, Wang YY, Cao LY, Wen CP. Effects of shikonin isolated from zicao on lupus nephritis in NZB/W F1 mice. Biol Pharm Bull 2009; 32: 1565-1570

30 Xiong J, Ni J, Hu G, Shen J, Zhao Y, Yang L, Shen J, Yin G, Chen C, Yu G, Hu Y, Xing $M$, Wan $R$, Wang $X$. Shikonin ameliorates cerulein-induced acute pancreatitis in mice. J Ethnopharmacol 2013; 145: 573-580

31 Bai GZ, Yu HT, Ni YF, Li XF, Zhang ZP, Su K, Lei J, Liu BY, Ke CK, Zhong DX, Wang YJ, Zhao JB. Shikonin attenuates lipopolysaccharide-induced acute lung injury in mice. J Surg Res 2013; 182: 303-311

32 Chiu SC, Tsao SW, Hwang PI, Vanisree S, Chen YA, Yang NS. Differential functional genomic effects of anti-inflammatory phytocompounds on immune signaling. BMC Genomics 2010; 11: 513

33 Li T, Yan F, Wang R, Zhou H, Liu L. Shikonin suppresses human T lymphocyte activation through inhibition of IKK $\beta$ activity and JNK phosphorylation. Evid Based Complement Alternat Med 2013; 2013: 379536

34 Assimopoulou AN, Papageorgiou VP. Radical scavenging activity of Alkanna tinctoria root extracts and their main constituents, hydroxynaphthoquinones. Phytother Res 2005; 19: 141-147

35 Jin $R$, Bai Y. Theoretical investigation of the radical scavenging activity of shikonin and acylshikonin derivatives. J Mol Model 2012; 18: 14011408

36 Nishizawa M, Kohno M, Nishimura M, Kitagawa A, Niwano Y. Presence of peroxyradicals in cigarette smoke and the scavenging effect of shikonin, a naphthoquinone pigment. Chem Pharm Bull 2005; 53: 796-799

37 Wang Z, Liu T, Gan L, Wang T, Yuan X, Zhang B, Chen H, Zheng Q. Shikonin protects mouse brain against cerebral ischemia/reperfusion injury through its antioxidant activity. Eur J Pharmacol 2010; 643: 211-217

38 Nam KN, Son MS, Park JH, Lee EH. Shikonins attenuate microglial inflammatory responses by inhibition of ERK, Akt, and NF- $k \mathrm{~B}$ : neuroprotective implications. Neuropharmacology 2008; 55: 819-825

39 Esmaeilzadeh E, Gardaneh M, Gharib E, Sabouni F. Shikonin protects dopaminergic cell line PC12 against 6-hydroxydopamine-mediated neurotoxicity via both glutathione-dependent and independent pathways and by inhibiting apoptosis. Neurochem Res 2013; 38: 1590-1604

40 Andrikopoulos NK, Kaliora AC, Assimopoulou AN, Papapeorgiou VP. Biological activity of some naturally occurring resins, gums and pigments against in vitro LDL oxidation. Phytother Res 2003; 17: 501-507

41 Yoshida LS, Kawada T, Irie K, Yuda Y, Himi T, Ikemoto F, Takano-Ohmuro $H$. Shikonin directly inhibits nitric oxide synthases: possible targets that affect thoracic aorta relaxation response and nitric oxide release from RAW 264.7 macrophages. J Pharmacol Sci 2010; 112: 343-351

42 Rajasekar S, Park da J, Park C, Park S, Park YH, Kim ST, Choi YH, Choi YW. In vitro and in vivo anticancer effects of Lithospermum erythrorhizon extract on B16F10 murine melanoma. J Ethnopharmacol 2012; 144: 335-345

43 Kretschmer N, Rinner B, Deutsch AJ, Lohberger B, Knausz H, Kunert O, Blunder $M$, Boechzelt $H$, Schaider $H$, Bauer $R$. Naphthoquinones from Onosma paniculata induce cell-cycle arrest and apoptosis in melanoma cells. J Nat Prod 2012; 75: 865-869

44 Min R, Zun Z, Min Y, Wenhu D, Wenjun $Y$, Chenping Z. Shikonin inhibits tumor invasion via down-regulation of NF- $\kappa$ B-mediated MMP-9 expression in human ACC-M cells. Oral Dis 2011; 17: 362-369

45 Yang H, Zhou P, Huang H, Chen D, Ma N, Cui QC, Shen S, Dong W, Zhang X, Lian W, Wang $X$, Dou QP, Liu J. Shikonin exerts antitumor activity via proteasome inhibition and cell death induction in vitro and in vivo. Int J Cancer 2009; 124: 2450-2459

46 Chang IC, Huang YJ, Chiang TI, Yeh CW, Hsu LS. Shikonin induces apoptosis through reactive oxygen species/extracellular signal-regulated kinase pathway in osteosarcoma cells. Biol Pharm Bull 2010; 33: 816824

47 Chen J, Xie J, Jiang Z, Wang B, Wang Y, Hu X. Shikonin and its analogs inhibit cancer cell glycolysis by targeting tumor pyruvate kinase-M2. Oncogene 2011; 30: 4297-4306

$48 \mathrm{Li}$ W, Liu J, Zhao Y. PKM2 inhibitor Shikonin suppresses TPA-induced mitochondrial malfunction and proliferation of skin epidermal JB6 cells. Mol Carcinogen 2012; DOI: 10.1002/mc.21988

49 Yingkun N, Lvsong Z, Huimin Y. Shikonin inhibits the proliferation and induces the apoptosis of human HepG2 cells. Can J Physiol Pharmacol 2010; 88: 1138-1146
50 Han W, Li L, Qiu S, Lu Q Pan Q Gu Y, Luo J, Hu X. Shikonin circumvents cancer drug resistance by induction of a necroptotic death. Mol Cancer Ther 2007; 6: 1641-1649

51 Andújar I, Recio MD, Giner RM, Ríos JL. Traditional Chinese medicine remedy to jury: the pharmacological basis for the use of shikonin as an anticancer therapy. Curr Med Chem 2013; 20: 2892-2898

52 Huang C, Luo Y, Zhao J, Yang F, Zhao H, Fan W, Ge P. Shikonin kills glioma cells through necroptosis mediated by RIP-1. PLoS ONE 2013; 8: e66326

53 Piao JL, Cui ZG, Furusawa Y, Ahmed K, Rehman MU, Tambuchi Y, Kadowaki M, Kondo T. The molecular mechanisms and gene expression profiling for shikonin-induced apoptotic and necroptotic cell death in U937 cells. Chembiol Interact 2013; DOI: 10.1016/j.cbi.2013.06.011

54 Ahn J, Won M, Choi JH, Kim YS, Jung CR, Im DS, Kyun ML, Lee K, Song KB, Chung KS. Reactive oxygen species-mediated activation of the Akt/ ASK1/p 38 signaling cascade and p21(Cip1) downregulation are required for shikonin-induced apoptosis. Apoptosis 2013; 18: 870-881

55 Zhang $B$, Chen $N$, Chen $H$, Wang Z, Zheng $Q$. The critical role of redox homeostasis in shikonin-induced HL-60 cell differentiation via unique modulation of the Nrf2/ARE pathway. Oxid Med Cell Longev 2012; 2012: 781516

56 Wang H, Wu C, Wan S, Zhang H, Zhou S, Liu G. Shikonin attenuates lung cancer cell adhesion to extracellular matrix and metastasis by inhibiting integrin $\beta 1$ expression and the ERK1/2 signaling pathway. Toxicology 2013; 308: 104-112

57 Haghbeen K, Pourmolaei S, Mareftjo MJ, Mousavi A, Akbari Noghabi K, Hosseini Shirazi F, Meshkat A. Detailed investigations on the solid cell culture and antimicrobial activities of the Iranian Arnebia euchroma. J Biomed Biotechnol 2011; 2011: 165852

58 Ding X, Yin B, Qian L, Zeng Z, Yang Z, Li H, Lu Y, Zhou S. Screening for novel quorum-sensing inhibitors to interfere with the formation of Pseudomonas aeruginosa biofilm. J Med Microbiol 2011; 60: 18271834

59 Shen CC, Syu WJ, Li SY, Lin CH, Lee GH, Sun CM. Antimicrobial activities of naphthazarins from Arnebia euchroma. J Nat Prod 2002; 65: 1857 1862

60 Li HM, Tang YL, Zhang ZH, Liu CJ, Li HZ, Li RT, Xia XS. Compounds from Arnebia euchroma and their related anti-HCV and antibacterial activities. Planta Med 2012; 78: 39-45

61 Kuo HM, Hsia TC, Chuang YC, Lu HF, Lin SY, Chung JG. Shikonin inhibits the growth and $\mathrm{N}$-acetylation of 2-aminofluorene in Helicobacter pylori from ulcer patients. Anticancer Res 2004; 24: 1587-1592

62 Sasaki $K$, Abe $H$, Yoshizaki $F$. In vitro antifungal activity of naphthoquinone derivatives. Biol Pharm Bull 2002; 25: 669-670

63 Miao H, Zhao L, Li C, Shang Q Lu H, Fu Z, Wang L, Jiang Y, Cao Y. Inhibitory effect of shikonin on Candida albicans growth. Biol Pharm Bull 2012; 35: 1956-1963

64 Michaelakis A, Strongilos AT, Bouzas EA, Koliopoulos G, Couladouros EA Larvicidal activity of naturally occurring naphthoquinones and derivatives against the West Nile virus vector Culex pipiens. Parasitol Res 2009; 104: 657-662

65 Ali A, Assimopoulou AN, Papageorgiou VP, Kolodziej H. Structure/antileishmanial activity relationship study of naphthoquinones and dependency of the mode of action on the substitution patterns. Planta Med 2011; 77: 2003-2012

66 Chen X, Yang L, Zhang N, Turpin JA, Buckheit RW, Osterling C, Oppenheim $J J$, Howard OM. Shikonin, a component of Chinese herbal medicine, inhibits chemokine receptor function and suppresses human immunodeficiency virus type 1 . Antimicrob Agents Chemother 2003; 47: 2810-2816

67 Min BS, Miyashiro H, Hattori M. Inhibitory effects of quinones on RNase $\mathrm{H}$ activity associated with HIV-1 reverse transcriptase. Phytother Res 2002; 16: S57-S62

68 Gao H, Liu L, Ou ZY, Wei FX, Wang SQ, Chen G, Oin L, Jiang FY, Wang YC, Shang L, Gao CY. Anti-adenovirus activities of shikonin, a component of Chinese herbal medicine in vitro. Biol Pharm Bull 2011; 34: 197-202

69 Karayannopoulou M, Tsioli V, Loukopoulos P, Anagnostou TL, Giannakas $N$, Savvas I, Papazoglou LG, Kaldrymidou E. Evaluation of the effectiveness of an ointment based on Alkannins/Shikonins on second intention wound healing in the dog. Can J Vet Res 2011; 75: 42-48

70 Hsiao CY, Tsai TH, Chak KF. The molecular basis of wound healing processes induced by Lithospermi Radix: a proteomics and biochemical analysis. Evid Based Complement Alternat Med 2012; DOI: 10.1155/ $2012 / 508972$ 
71 Andújar I, Ríos JL, Giner RM, Recio MC. Shikonin promotes intestinal wound healing in vitro via induction of TGF- $\beta$ release in IEC-18 cells. Eur J Pharm Sci 2013; 49: 637-641

72 Gwon SY, Ahn JY, Chung CH, Moon BK, Ha TY. Lithospermum erythrorhizon suppresses high-fat diet-induced obesity, and acetylshikonin, a main compound of Lithospermum erythrorhizon, inhibits adipocyte differentiation. J Agric Food Chem 2012; 60: 9089-9096

73 Lee H, Kang R, Yoon Y. Shikonin inhibits fat accumulation in 3 T3-L1 adipocytes. Phytother Res 2010; 24: 344-351

74 Lee H, Bae S, Kim K, Kim W, Chung SI, Yang Y, Yoon Y. Shikonin inhibits adipogenesis by modulation of the WNT/ $\beta$-catenin pathway. Life Sci 2011; 88: 294-301
75 Kamei R, Kitagawa Y, Kadokura M, Hattori F, Hazeki O, Ebina Y, Nishihara T, Oikawa S. Shikonin stimulates glucose uptake in 3 T3-L1 adipocytes via an insulin-independent tyrosine kinase pathway. Biochem Biophys Res Commun 2002; 292: 642-651

76 Nigorikawa K, Yoshikawa K, Sasaki T, Iida E, Tsukamoto M, Murakami H, Maehama T, Hazeki K, Hazeki O. A naphthoquinone derivative, shikonin, has insulin-like actions by inhibiting both phosphatase and tensin homolog deleted on chromosome 10 and tyrosine phosphatases. Mol Pharmacol 2006; 70: 1143-1149

77 Öberg AI, Yassin K, Csikasz RI, Dehvari N, Shabalina IG, Hutchinson DS, Wilcke M, Östenson CG, Bengtsson T. Shikonin increases glucose uptake in skeletal muscle cells and improves plasma glucose levels in diabetic Goto-Kakizaki rats. PLoS One 2011; 6: e22510 